\section{Primary
hydatidosis of the thyroid gland: a case report}

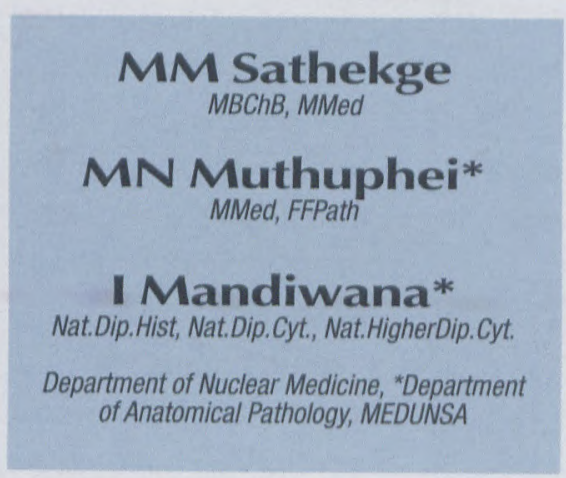

$\mathrm{E}$ chinococcosis, or hydatidosis, has a characteristic geographic distribution, occurring most frequently in sheep-rearing regions such as the Mediterranean countries, Oceania and South Africa. 'The cysts are most commonly seen in the liver and lungs, although involvement of other organs is possible but rare. ${ }^{2}$

Hydatid disease in the head and neck region is rare and to our knowledge no English report of such cases in the thyroid gland has been published. The aim of this report is to discuss the importance of the combination of scintigraphy and ultrasonography prior to fine needle aspiration (FNA) in the diagnosis and management of swellings of the thyroid gland.

\section{Case report}

A 29-year-old woman presented with a single thyroid nodule of 6 months duration. For the past 3 weeks the swelling was noticed to be increasing in size. Progressive swelling was associated with increased pressure, mild pain on palpation of the mass and dysphagia.

Physical examination at the time of presentation revealed a $6 \mathrm{~cm}$ diameter soft mass in the left mid neck which was mildly tender on palpation. The mass was mobile during swallowing.

Laboratory evaluation including complete blood count, erythrocyte sedimentation rate, T3, T4, TSH and LFT were all normal. Thyroid scintigraphy with Tc-99m pertechnetate revealed a focal area of photopenia in the upper zone of the left thyroid lobe on both flow and static images (Figure 1). The scintiscan findings correlated well with the clinical impression. Ultrasonography was not performed because the thyroid swelling was not 


\section{Primary hydatidosis of the thyroid gland: a case report}

\section{from page 13}

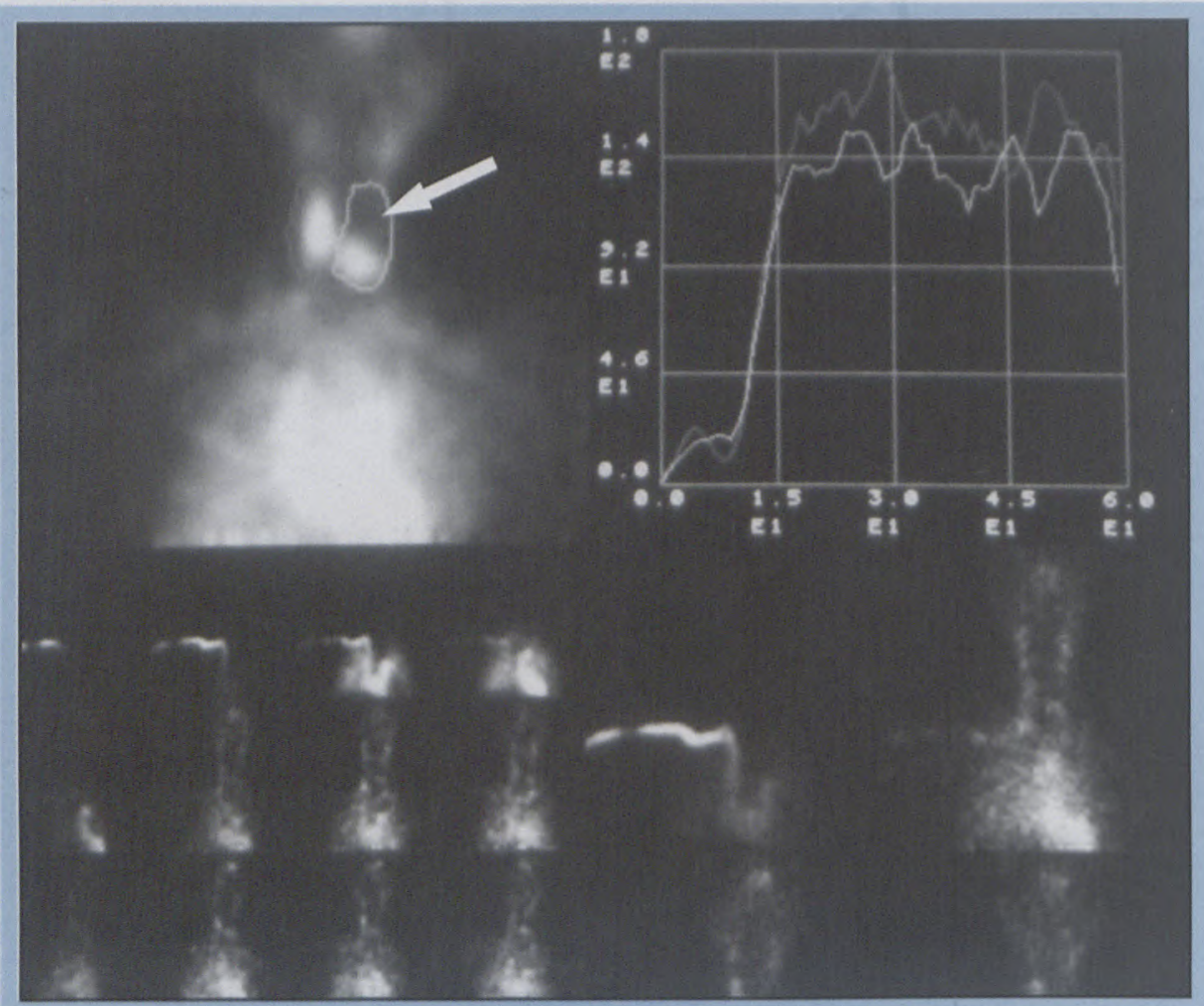

Figure 1: (A) Perfusion scan: a photopenic area (arrow) in the left upper zone.

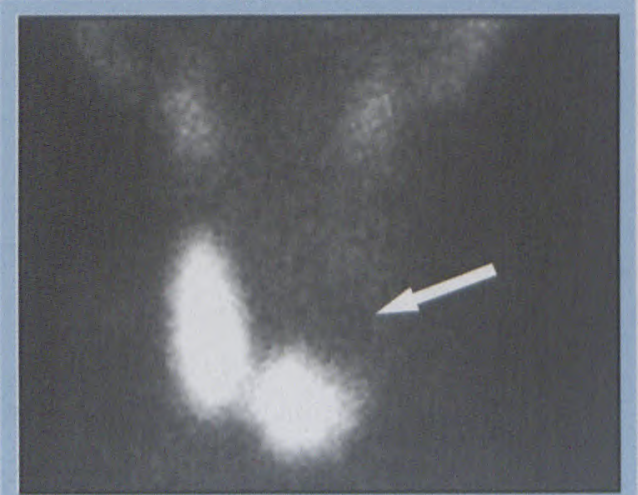

Figure 1(B): Uptake scan: focal area of photopenia in the upper zone (arrow) of the left lobe persists.

thought to be a cyst on clinical or scintigraphic analysis. Cytology was undertaken based on the scintigraphic results. This showed multiple calcified bodies and very few cells. There were groups of scolices resembling those of Echinococcus granulosus (Figure 2).

The resected specimen showed thyroid partially replaced by a large cyst with a gelatinous appearance.

Histological examination revealed a parasitic membrane with degenerated scolices and hooklets.

Figure 2: A scolex of Echinococcus granulosus.

Extensive dystrophic calcification was present (Figure 3). The surrounding thyroid tissue was severely inflamed with many foreign body giant cells being seen. A diagnosis of hydatid cyst was made.

Postlobectomy thyroid scintigraphy and ultrasound showed no residual thyroid tissue on the left side.

\section{Discussion}

An unusual location for hydatid disease in the thyroid gland has to our knowledge not been presented before. Ultrasonography and CT usually easily detect hydatid disease, with the exception of hydatid cysts in uncommon sites of the body. ${ }^{3}$ The diagnosis of the disease prior to surgery is crucial so as to avoid spillage of the parasitic cyst's contents.

In our patient, some diagnostic difficulty was experienced as ultrasonography was not performed. It is generally agreed that the routine use of ultrasound in the evaluation of thyroid nodules is not cost effective. ${ }^{4}$ However the patient underwent thyroid scintigraphy because it is routine in all our patients with thyroid disease. The essential objective of thyroid scanning is to identify whether a nodule is hypofunctioning, not only because this is the most likely finding, but because a solitary nodule is associated with an increased probability of malignancy. ${ }^{4}$ The quoted incidence of thyroid malignancy varies considerably in

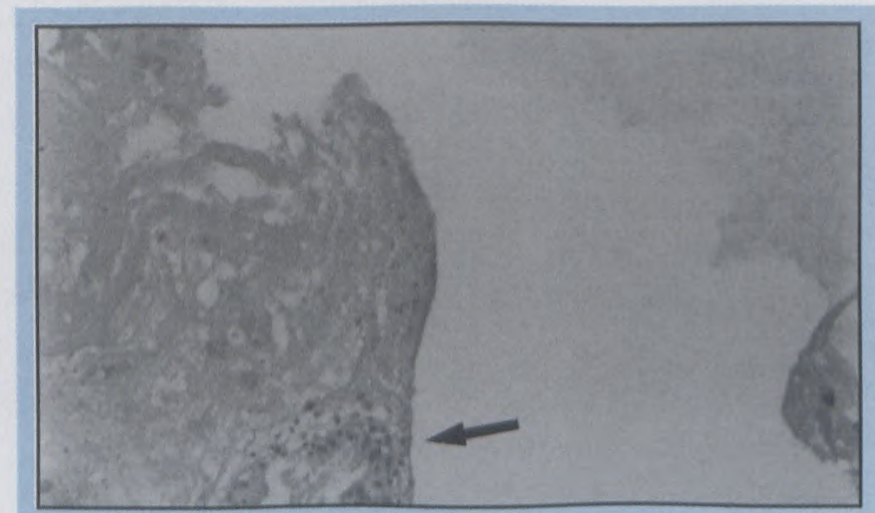

Figure 3: A section from the cyst wall showing a degenerated scolex (arrow). the literature, but is such that it should be excluded in every nodule shown to be nonfunctioning. In our 